\title{
The Psychological Effects of Urban Design
}

\author{
By Jonathan Monfries
}

\begin{abstract}
Regardless if we are aware of it or not, our mental wellbeing is being directly affected by our surroundings - the built environment. Certain styles of buildings, the layout of streets, and the provision of green spaces are altering our psychology subconsciously. There are several ways in which cities can encourage better mental wellbeing by considering better urban design. During an age where it is becoming increasingly important to comprehend the impacts of poor mental health, an opportunity utilizing urban planning can help contribute to a healthier mental state overall. This paper seeks to present the various ways cities can utilize urban design to help improve the mental health of its citizens, and a case study from Tokyo, Japan is analyzed. The paper is written from an urban planning perspective; however, it also includes a brief introduction to the psychological background on how exactly our minds are affected by the built environment.
\end{abstract}

"We shape our buildings, and afterwards our buildings shape us."

- Winston Churchill

\section{Introduction}

Whether it be in the form of taking transit, walking downtown, or simply the daily commute to work, we engage with the city we live in every day. We see buildings, spaces, streets, and we interact with other people. While we may not be consciously aware of it, the environment that we occupy can influence our behaviours and our mental state. As mental health continues to be an important societal consideration, we must ask ourselves: are there every day, recurring objects, signs, or environments that are influencing our mental health? This paper analyzes the research and theories behind the concept of how urban design can affect our psychology and mental wellbeing. Additionally, this paper will discuss certain aspects of cities that reinforce the importance of considering the effects of the built environment on mental health. Finally, a case study about Tokyo, Japan will be analyzed to illuminate the beneficial impacts that a well-designed city can have on our mental state. In an increasingly urbanized world, it has become imperative that we consider how urban design in cities affects our mental wellbeing.

\section{The Current State of Urban Life in Cities and How It Affects our Psychology}

Cities are places that provide people with occupations, recreational activities, environmental space, amenities, public art, architecture, and open spaces to encourage interaction between citizens. However, several studies indicate that urban life is associated with an increased incidence of numerous mental health problems. For instance, a study by Peen, Schoevers, and Dekker (2010) found that living in cities is associated with increases in mood disorders (by up to $39 \%$ ), increases in anxiety disorders (by up to $21 \%$ ), doubling the risk of schizophrenia, and increases in cocaine and heroin addictions. While these effects from city living can be attributed to a variety of factors, it is clear that city life can negatively affect our mental health. Social and political issues 
have effects on these matters as well, but as urban planners and architects, a certain level of responsibility should be taken to ensure that our buildings, streets, and public spaces are supporting positive mental health in citizens. Advancing the state of global mental health was addressed by the World Health Organization's Comprehensive Mental Health Action Plan, and with $68 \%$ of the world's population projected to be living in urban areas by 2050 (UN, 2018), achieving positive mental health is a global issue.

\section{In What Ways Does the Built Environment Affect Psychology?}

The connection between psychology and the built environment can be traced back to the origins of architectural design and the work of the Roman architect Vitruvius. De Architectura represented a seminal work for the profession, where Vitruvius argued that good building design should reflect the form and proportions of the human body. Our brains are emotionally affected by other faces due to areas of the brain that are specialized for recognition of facial expressions, and these processes occur at an incredibly high speed, without our conscious awareness (Ellard, 2015). Based on the concept that we have an innate ability to respond emotionally when viewing a face, it is reasonable to believe that a similar emotional response could occur when we view an intriguing building or open space design. In fact, a study by Ostwald et al. (2012) analyzed how certain buildings can evoke this facial recognition effect by utilizing a variety of camera software. The authors found that certain buildings, such as Frank Lloyd Wright's Robie House, read as "angry", owing to its very restrictive and somewhat brutalist design. This represents a sort of semiotics in that the house's elements can represent symbols of anger and therefore evoke an emotional response in those who look at the building. This idea can be applied to a broader example of the built environment in general and how combinations of buildings and open spaces lead to a variety of emotions evoked in the viewer. However, the presence of a variety of design in the downtown of a city can lead to emotional distress, especially when an individual is confronted with different design elements and conflicting emotional responses. These conflicting emotions can aggravate an individual's anxiety or other mental health issues. Therefore, it is important to consider the design of buildings, streetscapes, and open spaces, and how they can evoke emotional responses in our minds.

The ways in which urban design affects psychology and our mental health have been found to consist of seven major factors, building on preliminary research from the Centre for Urban Design and Mental Health (UDMH) (2017). Some examples speak to specific instances of design, whereas others focus on the psychological aspects of social interaction. Still, others discuss the effects of the urban society as a whole, such as the perceived sense of safety and the state of economics and affordability in a city. These factors are discussed more in depth within the following sections of this paper.

\section{Green Spaces, Access to Nature, and Opportunities for an Active Lifestyle}

Often it is true that improving an individual's physical health will benefit their mental health as well. Including a sufficient amount of public spaces in cities allows for easy to access space that can promote active lifestyles. A study by Roe (2016) supports this claim, as it was found that urban green space has a beneficial effect on individuals with mental health problems such as stress, anxiety, and depression. Improving the presence of trees, pocket parks, and even outdoor exercise machines, particularly in a city's downtown area, can have a significant impact on improving people's emotions, behaviours, and overall mental wellbeing. Spaces such as these encourage people to talk to each other, increasing social interaction, as well as provide better walkability between their destinations. A major goal for our cities is to ensure that people have "consistent, regular exposure to urban nature" throughout daily commutes and routines (UDMH, 2017). This includes natural areas along streets (such as trees and bushes), pocket parks near bus stops, and green spaces 
that are viewable from office windows to positively benefit the office environment as well. These green spaces need to be regularly maintained to prevent them from falling into a state of disrepair, where such a case would lead to a negative effect on mental health.

Public green spaces can also be utilized as spaces for physical activity. Urban parks get a significant amount of use because citizens can use them as an escape from the bustling city life. Successful examples include New York's Central Park, Vancouver's Stanley Park, and Edmonton's North Saskatchewan River Valley. However, it does not have to be limited to large scale parkland. Small pocket parks located in previously vacant lots downtown can serve as an outdoor gym allowing people to include exercise in their daily routines, or even during a lunch break. Exercise increases serotonin in the brain, which can help with improving people's moods and emotions (UDMH, 2017).

Urban design can also allow for more physical activity by providing more bicycle parking so that more people use active transportation to get to work. Including the provision of bike lanes and ensuring that sidewalks are wider and buffered from roads creates a sense of safety and ease for those who choose to walk to their destination rather than drive.

\section{Encouraging Social Interaction and Safety in the City}

As has been alluded to previously in this paper, social interaction is a significant element in ensuring good mental wellbeing. In terms of urban design, social interaction is often achieved by concentrating groups of people in a single node or landmark so that it is suggested they interact with each other. Strategies for urban design to accommodate this can include higher density development, so that more people are concentrated in an area. Additionally, developments with a mix of uses allow for consistent foot traffic on the street rather than strictly an office building street frontage that would only see traffic during the morning, lunch, and evening commutes. However, it is not just a matter of providing numerous open spaces for the public to socially interact. A study by Francis, Wood, Knuiman, and Giles-Corti (2012) in Western Australia found that the quality, not the quantity of open spaces was conducive to better mental health, and a single, well-designed open space proved to be more beneficial than multiple poorly-designed open spaces. Such poorlydesigned open spaces would be significantly less likely to foster social interaction as less people would be inclined to spend time in these spaces.

As demonstrated from the shortfalls of Le Corbusier's infamous Pruitt-Igoe housing project (Heathcott, 2012), it is important to ensure that social housing is located near public space to encourage physical activity as well. Locating developments such as these near recreation facilities, parks, and fitness centres allows for easy access to physical exercise services. Additionally, taking into consideration the proximity of amenities such as grocery stores, schools, and libraries encourages residents to consider active transportation due to these services.

A perceived sense of safety is another significant factor in ensuring mental wellbeing in cities. Safety can relate to vehicle traffic, the feeling of being lost, environmental pollutants and exhaust, and crime (UDMH, 2017). In order to retain a healthy mental state, people need to feel a sense of security. As was alluded to in semiotic theory, various elements of disrepair can be a sign for negative feelings, and the fact that potentially a crime had taken place there. Therefore, a sense of being unsafe could trigger anxiety or stress in those who do not feel secure. (CEBCP, 2017). As such, it is important for urban design to consider streets that promote good visibility, and walkways where you are able to see far ahead to where you are going. This helps to prevent the feeling of being lost or isolated, and also provides a subconscious route for escape in case of adversity, rather than feeling trapped. Taking a people-oriented approach to design would help encourage safety when considering the frontages of buildings on sidewalks, as well as the buffers from active vehicular traffic on an arterial roadway. Ensuring that spaces and buildings are designed to limit dark corners 
will prevent areas that can harbour increased criminal activity. As suggested by Kevin Lynch in Image of the City (1960), the presence of landmarks helps for people to identify where they are, and where they have relocated in relation to that landmark. This can help with the feeling of being lost in a city (Lynch, 1960).

\section{Transportation, the Environment, and the Economy}

Our psychology is significantly influenced by our daily commutes through the city. It can put us into a certain mood or set of behaviours both to and from work, for example. Our surroundings are constantly influencing our minds. Ensuring a city provides good transportation and connectivity is beneficial by making commutes less stressful (particularly with using public transit) and improving accessibility to amenities and services. This efficiency of transportation can help to reduce issues of stress and anxiety because it makes urban living significantly easier. Additionally, public transportation is a more environmentally friendly way to commute, reducing contributors of air pollution. Poor air quality has numerous physical health effects, but the provision of street trees and green spaces to naturally filter air, as well as directing building exhaust away from the street and implementing "idle-free" zones for traffic can all contribute to better overall health and wellbeing. Finally, economic disparity can have a significant effect on one's psychology. Neighbourhoods with high concentrations of impoverished people are often also highly underserved in terms of services and amenities, and neglected for infrastructure upgrades. Urban design needs to help ensure that these blighted areas are designed for its users, particularly ensuring sidewalks are wider and bike lanes are provided due to the significant amount of people that do not drive and can only walk or bike. People who live in poorer neighbourhoods are likely to have more stress and frustration in their lives, and therefore how they get around their neighbourhood can have a significant impact on increasing their existing stresses.

\section{Tokyo Case Study}

Tokyo, Japan is a large metropolitan area with a population of almost 38 million people (WPR, 2017). Tokyo is a particularly interesting case for analyzing urban design and its impacts on mental health because the awareness and education about mental health there is not at the same state as it is in Canada. Any forms of mental health issues are often assumed to be nothing more than an illness (McCay, Suzuki, \& Chang 2017). While there are less mental health cases in Japan compared to Western countries, it could be due to factors including the lack of care and services provided for those who do decide to speak out about their mental illness, and/or decreased awareness or acknowledgement of mental health conditions. The city has also been known to have a stressful work culture, with many instances of people having to consistently work longer overtime hours, with high levels of overall stress, and poor food security (McCay et al., 2017). However, Tokyo has some planning policies in place that do address some mental health initiatives indirectly, although the conversation about the importance of the issue needs more support. This case study will analyze Tokyo's provision of some of the most significant aspects of a city that can improve mental wellbeing which have been discussed in this paper.

\section{Providing for Green Spaces and Physical Activity in Tokyo}

In terms of green space, Tokyo has an abundance of natural areas within the city, however not all are managed and conserved as well as they should (TMG, 2017). Many public green spaces in Tokyo are not open to the public because they are either located on private property or are on lands for a local temple. This creates an issue where more green space is not necessarily a requirement for the city, but simply better provisioning and conservation of these green spaces to ensure that the public has access to them. In a downtown cityscape where people want to escape to green spaces to help calm their stresses and anxieties, publicly accessible green spaces are important so that all individuals 
can enjoy the benefits. Understandably, if green space becomes limited to private groups solely, the public would not have access and therefore be unable to utilize public space for calming and alleviating anxiety. Fortunately, the Tokyo Metropolitan Government (TMG) has been starting to address the need for green spaces. A variety of plans such as the Green Master Plan, Greenery Doubling Plan, and a Green Tokyo Plan have been implemented and while these plans may not explicitly mention the benefits to mental health, they will indirectly benefit the cause. For example, the plans suggest that a "green road network" is established where trees and roadside planters are installed to create a "green buffer" from traffic (TMG, 2007). The plans also suggest that an additional 1,000 ha of green space is provided in the metropolitan area. While some of the goals are ambitious, it is a good initial attempt at ensuring more public green space is provided in the city.

Tokyo experiences an advantage when it comes to providing opportunities for physical activity, in that the vast majority of citizens do not commute to work by vehicle, or even own a car for that matter. Therefore, residents of Tokyo can get to their destinations either by active transportation, or by walking to access a public transportation node. Additionally, the TMG's Bureau of Social Welfare and Public Health has started an initiative to encourage more walking, and specifically describes how walking can contribute to a significant change in mood and allow for stress release (McCay et al., 2017). Outdoor exercise areas already exist in Tokyo throughout the downtown core, allowing for people to exercise anytime throughout the day at no cost, making it accessible to everyone. As well, Tokyo has a significant number of cyclists (Kidd, 2015). Although regular commuters do not often take bicycles since most corporations are required by legislation to insure their employees during their commute la high cost to absorb, as biking is not necessarily the safest method of transportation in the city), cycling is increasingly popular with women and particularly mothers who ride a "mamachari", which is a commuter bike with space for groceries and children
(McCay et al., 2017; Kidd, 2009). Additionally, the TMG is taking significant actions to prepare its city for the Olympic games in 2020. This includes several elements that will ensure Tokyo is designed to be more engaging and supportive of physical and mental wellbeing (Kidd, 2015). With regards to active transportation, this includes the provision of over $400 \mathrm{~km}$ of safe cycling routes, which are similar to bike lanes. The only issue with the efforts to design new bike lanes is the fact that many locals feel the efforts are only beneficial to tourists and visitors, and if the network is actually realized, it would only help the commutes of residents located nearest the core (Kidd, 2015). Therefore, it is important for Tokyo to ensure that it utilizes the Olympics as an opportunity to improve various means of infrastructure that can benefit both the tourism surge as well as its local residents.

\section{Encouraging Safety and Social Interaction in Tokyo}

Tokyo has an issue of restricting access to many of its public spaces. Public spaces are essential nodes of activity and engagement that can help foster social interaction, which is directly beneficial to better mental health and wellbeing. The city also lacks what many Western and European countries have: town squares (McCay et al., 2017). This creates a major issue because Tokyo is such a developed city, making it very difficult to design these spaces in an already developed downtown core. Cities throughout European countries in most cases had these town squares since the inauguration of the city, and then development occurred around it. Therefore, an emerging trend in Tokyo is the provision of social spaces that are indoors. Indeed, this contradicts the benefits to mental health by providing outdoor natural areas, but in an extensively developed and congested city like Tokyo, all that is left for change may be indoors. Such spaces would be provided in office tower lobbies, to provide for a better environment for employees as well as the public, who are allowed to spend time and interact with each other in the office building. Due to the long working hours often observed 
in Tokyo, such spaces inside office buildings would have a beneficial impact on the stressful lives of a typical office worker. In addition, indoor spaces provide a refuge from inclement weather. In fact, because Tokyo already has such a low crime rate, the most significant safety risk is the adverse natural disasters, primarily earthquakes and tsunamis (McCay et al., 2017). In this case, it is important that spaces for social interaction are especially designed to withstand the elements and allow for people to not only easily access the space, but also ensure that it is identifiable, easy to locate, and does a sufficient job to keep those who are inside of it safe.

\section{Transportation and Accessibility in Tokyo}

Tokyo has an extensive transit system that, while overcrowded, is affordable and easily accessible by the public. A key element of designing for better mental health relates to how we commute between our destinations. Tokyo's transit system is found to be very reliable and consistent, and because of its low price, people of all classes and stages of life are easily able to access it (McCay et al., 2017). In addition to a sufficient transit system, more improvements are planned or have already been recently completed in anticipation of the 2020 Olympic games. This creates opportunities for Tokyo to make significant improvements which can positively influence the psychology of both citizens and visitors. The provision of more bike lanes and safer sidewalks contribute to less-stressful modes of transportation for the public to get to their destinations with ease.

One limitation of Tokyo's infrastructure is their use of the waterfront. Providing numerous opportunities for the public to easily access the waterfront is critical to allow for an escape from the stresses and noise of the city. One would expect Tokyo to have a strong presence of vehiclelimited promenades and boardwalks along the waterfront; however, a quick observance in Google Earth would show that this is not the case. Therefore, it is important for the TMG, as well as planners and architects, to increase efforts to engage the public with the waterfront and provide more pedestrian-only promenades.
In anticipation of the 2020 Olympics, it would likely be seen as highly beneficial for the city to provide these areas that benefit the mental health of visitors and tourists alike.

\section{Conclusion}

Our day-to-day interactions with the cities we live in have a significant effect on our everyday life. The meanings we deduce from building designs or public spaces can in turn affect our psychology and how we feel. Urban design can evoke both positive and negative emotions in us, affecting our mood and behaviours. Our psychology can be influenced by several urban aspects, such as green spaces, areas for social interaction, safety, transportation, and even economic factors. Mental health is growing into a significant issue at present, and an important consideration for this issue is accepting that recurring objects, signs, or environments making up a city's urban design can influence our mental health. By analyzing the research and theories behind the concept of urban design and psychology, it has been observed that there is a very real connection between design and mental health of the citizens living and working in urban centres. Therefore, whether it be in the way active transportation is considered, how green spaces are planned, or how communal gathering spaces are envisioned - in a world facing high rates of growth and urban development, it is essential that we consider the psychological effects of how we design our cities.

\section{References}

Centre for Evidence-Based Crime Policy (CEBCP). (2017). Broken windows policing. Retrieved from https://cebcp.org/ evidence-based-policing/what-worksin-policing/research-evidence-review/ broken-windows-policing/.

Francis, J., Wood, L.J., Knuiman, M., \& GilesCorti, B. (2012). Quality or quantity? Exploring the relationship between public open space attributes and mental health in Perth, Western Australia. Social Science \& Medicine, 74(10), 1570-1577. https://doi. org/10.1016/i.socscimed.2012.01.032 
Heathcott, J. (2012). Plannng Note: Pruitt-Igoe and the Critique of Public Housing. Journal of the American Planning Association, 78(4), 450-451. https://doi.org/10.1080 /01944363.2012.737972

Kidd, B. (2009). Introducing the Mamachari. Tokyo by Bike. Retrieved from http://www. tokyobybike.com/2009/06/introducingmamachari.html

Kidd, B. (2015). Tokyo plans $400 \mathrm{~km}$ of safe cycling routes... because the Olympics. Tokyo by Bike. Retrieved from http://www. tokyobybike.com/2015/05/tokyo-plans400km-of-safe-cycling.html

Lynch, K. (1960). The Image of the City. Cambridge, MA: MIT Press.

McCay, L., Suzuki, E., Chang, A. (2017). Urban design and mental health in Tokyo: a case study. Journal of Urban Design and Mental Health, 3(4). Retrieved from https://www. urbandesignmentalhealth.com/journal-3--tokyo-case-study.html

Ostwald, M.J., Hong, K., Chalup, S. (2012). Pareidolia analysis of architecture: Reading the emotional expression of a building façade. Academy of Neuroscience for Architecture. Retrieved from http://anfarch. org/wp-content/uploads/2013/11/ OstwaldPoster.pdf

Peen, J., Schoevers, A.T., Dekker, J. (2010). The current status of urban-rural differences in psychiatric disorders. Acta Psychiatrica Scandinavica, 121(2), 84-93. https://doi. org/10.1111/i.1600-0447.2009.01438.x

Roe, J. (2016). Cities, Green Space, and Mental Well-Being. Oxford Research Encyclopedia of Environmental Science. https://doi.org/10.1093/ acrefore/9780199389414.013.93

The Centre for Urban Design and Mental Health (UDMH). (2017). How Urban Design Can Impact Mental Health. Retrieved from https://www.urbandesignmentalhealth. com/how-urban-design-can-impactmental-health.html
Tokyo Metropolitan Government. (2007). Basic Policies for the 10-Year Project for Green Tokyo. Retrieved from: https:// www.kankyo.metro.tokyo.jp/en/ attachement/10-year_project.pdf

United Nations World Urbanization Prospects. (2018). Population Division. Retrieved from https://population.un.org/wup/ Download/

World Population Review. (2017). Tokyo Population History. Retrieved from http:// worldpopulationreview.com/world-cities/ tokyo-population/ 